\title{
Galactomannan and Real-Time PCR in the diagnosis of invasive Aspergillosis: preliminary data
}

\author{
Cristina Pedrotti, Lucia Collini, Filippo Salvetti, Daniela Dachille, Marina Gaino, \\ Silvia D'Arcangelo, Danila Bassetti, Rosanna Predazzer, Patrizia Ober, Paolo Lanzafame \\ U.O. Microbiologia e Virologia - Ospedale S. Chiara di Trento
}

Key words: Invasive Aspergillosis, Galactomannan, Real-Time PCR

\section{Utilizzo della ricerca del galattomannano e della PCR Real Time per la diagnosi delle aspergillosi invasive: dati preliminari}

\section{SUMMARY}

The diagnosis of invasive aspergillosis is notoriously difficult. The standard culture-based methods have shown considerable limitations in performance. For this reason, non-culture methods have been increasingly employed for the diagnosis of invasive aspergillosis, and, among them, the methods based on Real-Time polymerase chain reaction (RT-PCR). In this study we assess the contribution in lowering diagnosis errors provided by the RT-PCR method when run alongside other methods.

We analyzed 23 biological samples, 14 serum samples, and 9 bronchoalveolar lavage samples (BAL) from 10 immunocompromised patients who were selected according to EORTC/MSG criteria (European Organization for Research and Treatment of Cancer/Mycoses Study Group). On the serum sample we searched the galactomannan (GM) (Platelia Aspergillus ${ }^{\circledR}$ ) and the fungal genome (MycAssay ${ }^{\top M}$ Aspergillus); the BAL samples were subjected also to the culture tests.

In II serum samples the results showed concordance between GM and RT-PCR tests, while in 3 samples we report discordance: 2 results were GM positive and RT-PCR negative, and I results GM negative and RT-PCR indeterminate. In 5 BAL samples the results showed concordance between the two methods, while 4 were GM positive and RT-PCR negative.

The data, although still preliminary, suggest an increased accuracy in the diagnosis of suspected invasive aspergillosis when employing both RT-PCR and GM tests given that the RT-PCR test eliminates the false positive results of the GM test.The PCR methods require, however, further applications of this type of diagnostic because of the severe limit given by the lack of standardization.

\section{INTRODUZIONE}

Le Aspergillosi Invasive (AI) sono micosi opportunistiche con una mortalità molto alta sia negli adulti (80-100\%) sia nei bambini (75\%). L'AI è un'infezione opportunistica che colpisce soggetti con vari gradi di immunodepressione, i pazienti con grave e prolungata neutropenia sono i più esposti ad alto rischio di infezione, anche se negli ultimi decenni si è registrato un marcato incremento dei casi in altri gruppi a rischio costituiti da soggetti trapiantati di organo solido, con patologie polmonari croniche ostruttive (BPCO) e sottoposti a terapie immunosoppressive $(5,13)$. Il tasso di morbidità e di mortalità associato $\mathrm{ad} \mathrm{AI}$, proporzionale all'aumento di questi gruppi a rischio, è in costante aumento ed è quindi di fondamentale importanza approntare prima possibile un'appropriata terapia antifungina in grado di salvare la vita del paziente.

I farmaci antifungini come il posaconazolo, il voriconazolo, l'itraconazolo e le echinocandine hanno migliorato notevolmente le opzioni tera- peutiche per il trattamento dell'aspergillosi invasiva, sebbene il risultato clinico favorevole per il paziente sia largamente influenzato dall'inizio precoce ed efficace della terapia.

I metodi tradizionali per la diagnosi di AI si basano sulla clinica o su esami diagnostici non specifici (imaging), spesso invasivi, che presentano evidenti limiti di sensibilità con il rischio di risultati tardivi e inconcludenti. La diagnosi precoce di quest'infezione opportunistica è notoriamente difficile e risulta essere ancora una vera e propria sfida soprattutto a causa degli esigui strumenti diagnostici a disposizione $(2,16,19)$, dove l'isolamento di Aspergillus da materiale delle basse vie respiratorie nei pazienti non neutropenici è, talvolta, il primo dato microbiologico di AI polmonare risultando un' evidenza piuttosto tardiva a causa dalla lenta crescita del fungo sui terreni di coltura (da 48 ore a 4 giorni dalla semina).

Il dosaggio del galattomannano (polisaccaride presente nella parete cellulare di Aspergillus e di Penicillium species) nel siero, ha dato prova di

\section{Corresponding author: Cristina Pedrotti}

U.O. Microbiologia e Virologia - L.go Medaglie d'Oro, 9 - 38I 23 Trento - Tel.: 046I-903984; Fax 046I-9036I5

E-mail: cristina.pedrotti@apss.tn.it 
essere utile per la diagnosi nei pazienti neutropenici dove la sua sensibilità può superare 1' $80 \%$, mentre nei pazienti non neutropenici la sensibilità cade anche sotto il $50 \%(3,4,8,9,10,14)$. Tali differenze di performance sono probabilmente dovute alle differenze nella patogenesi della malattia da aspergillo nei vari gruppi di ospiti (8). La determinazione del galattomannano nei campioni di lavaggio broncoalveolare (BAL) si è dimostrata molto più sensibile rispetto a quella nel siero (>80\%) (2), ma i campioni di BAL non sono sempre disponibili.

I metodi basati sulla reazione polimerasica a catena Real-Time (RT-PCR), sono in grado di accelerare la diagnosi di AI (6) e sembra possano avere un ruolo nel monitoraggio della terapia antifungina (15) ma sono limitati dalla mancanza di standardizzazione dei protocolli di estrazione ed amplificazione, dalle differenze nel DNA-target, e nei metodi usati per esaminare i prodotti amplificati (8). Per queste ragioni tali tecniche non sono comprese fra i criteri diagnostici che definiscono le AI da parte della European Organization for Research and Treatment of Cancer e il Mycoses Study Group (EORTC/MSG) (7).

In questo lavoro sono stati analizzati i percorsi diagnostici delle infezioni fungine invasive da Aspergillus, disponibili in un laboratorio di routi$n e$, e riferiti ad una popolazione a rischio rappresentata da pazienti ematologici e non, valutando l'utilità dei test molecolari (RT-PCR), per migliorare la sensibilità e la specificità del risultato microbiologico nella difficile diagnosi di AI e ridurre i tempi di diagnosi.

\section{MATERIALI E METODI}

Sono stati analizzati 23 campioni biologici, 14 di siero e 9 di BAL, provenienti da 10 pazienti adulti selezionati in base ai criteri stabiliti dal EORTC/MSG (2012) (7) per la definizione di micosi invasiva. Per 9 pazienti è stato possibile ottenere, oltre al campione di siero anche il BAL, per uno invece (paziente 10 della Tabella 1) il prelievo del BAL era troppo invasivo. In due pazienti, 6 e 7, sono stati eseguiti due prelievi a distanza di circa 15 giorni. Nel paziente 10 il prelievo di siero è stato ripetuto tre volte a distanza di 4 giorni uno dall'altro.

I campioni biologici di BAL, previa centrifugazione per $10^{\prime}$ a $3.000 \mathrm{~g}$ e utilizzo del pellet, sono stati esaminati al microscopio dopo colorazione di Gram e coltivati su terreni per funghi (Sabouraud Destrosio Agar). I terreni di coltura sono stati incubati a $37^{\circ} \mathrm{C}$ per $48 \mathrm{~h}$. I funghi filamentosi isolati sono stati identificati in base alle loro caratteristiche morfologiche (procedura standard).

I campioni biologici di siero e BAL sono stati indagati per la ricerca del galattomannano (GM) utilizzando il test immunoenzimatico (EIA) Platelia Aspergillus kit ${ }^{\circledR}$. Con questo test l'estrazione del GM avviene attraverso il calore $\left(100^{\circ} \mathrm{C} / 3^{\prime}\right)$ e centrifugazione a $10000 \mathrm{~g} / 10^{\prime}$, il sovranatante così ottenuto viene messo a contatto con anticorpi monoclonali adesi alla micro piastra, ricoperto con un coniugato composto da anticorpi monoclonali e perossidasi e incubato a $37^{\circ} \mathrm{C}$ per 90'. La presenza di GM nel campione genera un complesso a sandwich costituito da Ab monoclonale-GM-Ab monoclonale-perossidasi. La presenza di tale complesso viene evidenziata attraverso l'aggiunta del substrato contenente una soluzione cromogena che genera una reazione di colore blu. La reazione viene infine bloccata dall'aggiunta di una soluzione di $\mathrm{H}_{2} \mathrm{SO}_{4}$ che fa virare il colore dal blu al giallo. La lettura della densità ottica di ogni pozzetto viene fatta ad una lunghezza d'onda di $450 \mathrm{~nm}$ attraverso uno spettrofotometro. In ogni seduta, oltre ai campioni, si aggiungono le soluzioni dei controlli in doppio (positivo, negativo, di cut-off).

Dai valori della densità ottica misurata viene calcolato il valore Index per ogni campione secondo la formula seguente:

\section{$I=\frac{D . O \text { campione }}{\text { D.O media cut off }}$}

Il cut-off Index utilizzato per definire un campione positivo era, per il siero, $\geq 0.7$ per un singolo campione e $\geq 0.5$ per due campioni consecutivi (8), per il $\mathrm{BAL} \geq 1.0$ (20). L'esecuzione del test è completamente manuale.

Un'aliquota dei campioni così indagati è stata conservata a $-20^{\circ} \mathrm{C}$ e quindi processata per la ricerca del DNA di Aspergillus effettuata utilizzando il kit MycAssay ${ }^{\mathrm{TM}}$ Aspergillus (Myconostica, Cambrige, UK).

Il MycAssay ${ }^{\mathrm{TM}}$ Aspergillus è una tecnica di RTPCR per la rilevazione di Aspergillus DNA da campioni di siero, campioni provenienti dalle basse vie respiratorie, ma anche da altri materiali come il liquido cefalorachidiano. $\mathrm{E}$ in grado di rilevare 18 differenti specie di Aspergillus utilizzando sonde di ibridazione oligonucleotidiche a singolo filamento con struttura a sterm-loop (molecular beacons). Questo saggio utilizza come target il gene 18S rRNA e contiene un controllo interno di DNA ricombinante privo di omologie con la sequenza target per evitare i falsi negativi dovuti ad inibitori della PCR.

L'estrazione del DNA fungino è stata eseguita manualmente tramite colonnine cromatografiche QIAGEN. Il protocollo d'estrazione prevede una degradazione delle proteine di membrana e delle 
nucleasi cellulari attraverso l'utilizzo della proteinasi $\mathrm{K}$ (incubazione per $45^{\prime}$ a $90^{\circ} \mathrm{C}$ in un tubo da centrifuga di $100 \mu \mathrm{L}$ di campione, $20 \mu \mathrm{L}$ di proteinasi K, $220 \mu \mathrm{L}$ di PBS e $200 \mu \mathrm{L}$ del buffer contenente un sale caotropico), e quindi l'aggiunta di $220 \mu \mathrm{L}$ di etanolo per la precipitazione dell'acido nucleico. Il tutto viene poi trasferito in una colonnina contenente una membrana di silice che lega il DNA, i passaggi successivi di centrifugazione (8000rpm/1') e di lavaggi con etanolo e sali, mirano alla rottura del legame DNA-silice in modo da far precipitare il DNA nell'eluato per un volume finale di $300 \mu \mathrm{L}$. Per ogni campione di siero/BAL sono state eseguite due estrazioni separate così da ottenere un volume finale di $600 \mu \mathrm{L}$ tale da aumentare la probabilità di ricavare DNA fungino in soluzione e ridurre il numero di eventuali falsi negativi dovuti a una scarsa quantità di DNA.

Le concentrazioni di DNA nell'eluato sono state quantificate per mezzo di uno spettrometro di massa (NanoDrop Spectrophotometer ND-1000). L'acido nucleico così estratto e purificato è stato amplificato usando il MycAssay ${ }^{\mathrm{TM}}$ Aspergillus sulla piattaforma di SmartCycler (Chepheid, Sunnyvale, California, USA). $10 \mu \mathrm{L}$ di DNA estratto sono stati mescolati con i reagenti dell'amplificazione per un volume finale di $25 \mu \mathrm{L}$.

In tutto sono state condotte quattro sedute di amplificazione.

I risultati per ciascun campione sono stati riportati come negativi (campioni privi di amplificazione di DNA aspergillare e con controllo interno amplificato), positivi (campioni con amplificazione di DNA aspergillare) e indeterminati (campioni privi di amplificazione di DNA aspergillare e con fallimento dell'amplificazione del controllo interno). L'esame istologico (su biopsia polmonare), ritenuto test di riferimento, si è potuto eseguire solo per due pazienti (paziente 1 e paziente 7), per tutti gli altri l'esame istologico non è stato eseguito.

\section{RISULTATI}

Nella Tabella 1 sono riportati i risultati del GM e della RT-PCR riferiti ai sieri analizzati per ciascun paziente, mentre nella Tabella 2 sono riportati i risultati dell'esame microscopico e colturale, della ricerca del GM e del DNA su BAL per ciascun paziente.

Il GM è risultato essere positivo in 8 su 14 sieri analizzati. MycAssay ${ }^{\mathrm{TM}}$ Aspergillus è risultato positivo in 6 su 14 sieri analizzati. I due campioni non concordanti (con GM positivo e DNA negativo) appartengono ai pazienti 5 e 8 entrambi in terapia con piperacillina/tazobactam, riportata in letteratura come possibile causa di falsi positivi $(9,11)$.

I 6 sieri positivi con risultati confermati dai due metodi appartengono ai pazienti 6, 7 e 10: la ricerca è stata eseguita in doppio sia del GM che della RT-PCR per i pazienti 6 e 7, in triplo per il paziente 10 . Il paziente 6 e il paziente 7 con aspergillosi polmonare invasiva possibile, secondo i criteri di EORTC/MSG (7), presentano reperti microbiologici colturali il primo e istologici il secondo che avvalorano la concreta possibilità di un'infezione aspergillare a livello polmonare (da ritenersi dunque un'AI probabile). Per il paziente 10 , sotto stretto monitoraggio perché soggetto neutropenico ad alto rischio di sviluppo di AI, sono stati confrontati i dati di GM e RT-PCR di tre campioni sequenziali: il primo, GM negativo presenta un risultato in biologia molecolare indeterminato, il secondo e il terzo, GM positivi, si sono confermati positivi anche con la RT-PCR. Al momento della prima determinazione GM positiva il paziente non presentava ancora nessun segno di addensamento polmonare, che è stato rilevato nei giorni successivi fino a configurarsi come halo sign.

I risultati ottenuti dai due metodi sui 9 campioni di BAL sono stati meno concordanti. La ricerca del GM è risultata positiva in 6 su 9 campioni di BAL mentre MycAssay ${ }^{\mathrm{TM}}$ Aspergillus è risultato positivo in 2 su 9 campioni. Quattro campioni sono risultati non concordanti.

Il paziente 6 e il paziente 7 che presentano dati concordanti di positività per la ricerca di GM e di DNA fungino sono rispettivamente un paziente oncologico con metastasi di carcinoma del capocollo in trattamento chemioterapico, e un paziente con BPCO di grado clinico severo, già conosciuto e trattato chirurgicamente per aspergilloma polmonare (diagnosi istologica).

La positività del GM su BAL può essere espressione di colonizzazione delle basse vie aeree con Aspergillus, e per ridurre al minimo questo elemento confondente si è stabilito a 1 il valore Index di cut-off su BAL, anche se alcuni autori suggeriscono di alzarlo a 1.5 per aumentarne la specificità 8 , altri invece indicano un cut-off di 0.5 per aumentarne la sensibilità (18): due dei campioni GM positivi e DNA negativi presentavano un Index di 1.47 (paziente 1) e di 1.56 (paziente 4). Lo stesso problema di positività per colonizzazione delle vie respiratorie si ha con la ricerca del DNA fungino: il kit utilizzato ha cercato di ovviarvi usando un cut-off più restrittivo rispetto al siero, considerando negative tutte le curve di amplificazione salite a partire dal $36^{\circ}$ ciclo (su 38 cicli totali).

I pazienti 3 e 9 con un valore Index di GM su BAL molto elevato (4.5 e 8.4 rispettivamente) erano uno con BPCO end-stage, in terapia steroidea cronica, con insufficienza respiratoria acuta 
su un quadro di insufficienza respiratoria cronica (paziente 3); l'altro, in terapia immunosopressiva per patologia autoimmune, complicato da insufficienza renale cronica, con un addensamento polmonare persistente e resistente a terapia antibiotica (questo paziente aveva anche un riscontro microscopico positivo).

La negatività della RT-PCR sui BAL dei pazienti 3 e 9 è probabilmente riconducibile a inibizione da eccesso di DNA. Non è stato possibile verificare l'ipotesi ripetendo i test dopo diluizione in quanto non ancora chiaro, in base alle indicazioni della ditta produttrice, se la diluizione deve essere effettuata sul campione intero o dopo centrifugazione o sul DNA estratto.

L'esame microscopico dei campioni ha evidenziato 1 positivo su 9 campioni (paziente 9), e anche l'esame colturale è risultato positivo (Aspegillus fumigatus) in un campione su nove (paziente 6) (Tabella 2).

E da notare l'assoluta negatività microscopica e colturale del BAL del paziente 7 , unico del gruppo appartenente alla categoria con un riscontro istologico positivo da biopsia polmonare.

\section{DISCUSSIONE}

Il GM è un importante strumento diagnostico nella gestione di pazienti a rischio di AI e la sua presenza nel siero è considerata da EORTC/MSG uno dei criteri diagnostici nei pazienti oncologici (7). Nei pazienti neutropenici che hanno una relativamente alta probabilità a-priori $(>5-10 \%)$ di sviluppare AI è raccomandato il monitoraggio dei livelli di GM nel siero ogni 3-4 giorni. Un esempio di quanto sia importante il monitoraggio in questi soggetti ci viene dal paziente 10 , il cui la positività del GM nel siero ha preceduto la comparsa dei sintomi polmonari. In questo paziente il risultato indeterminato della RT-PCR sul primo campione GM negativo non ha permesso di verificare se il test molecolare sarebbe stato utilie per una diagnosi più precoce.

La gran parte degli studi, inoltre, indicano come l'andamento dei livelli di GM nel siero in pazienti sotto terapia antifungina sia predittivo della prognosi, e come la persistenza del GM, nonostante la terapia, sia un segno prognostico negativo tale da imporre una rivalutazione clinica. Purtroppo la specificità del GM è limitata da falsi positivi causati da numerosi fattori (9-11), tra questi vi è la cross-reattività con antibiotici beta-lattamici, che molto probabilmente spiega la positività dell'Index GM nel siero dei pazienti 5 e 8 . L'affiancamento di un altro metodo di ricerca rapido come la RT-PCR, permette di eliminare i falsi positivi intrinseci al polisaccaride galattomannano.
Nonostante l'esiguo numero di campioni esaminati in questo studio, la tecnica molecolare di ricerca del DNA fungino ha dimostrato una bassa performance sui campioni costituiti da broncolavaggio alveolare: sono sicuramente da approfondire e standardizzare le tecniche di estrazione dell'acido nucleico fungino (17) e da definire la diluizione di campioni con quantità di acido nucleico tale da inibire la reazione di amplificazione come è probabilmente accaduto per il $\mathrm{BAL}$ dei pazienti 3 e 9 .

Poiché la ricerca del GM nel siero di pazienti non ematologici è gravata da una bassa sensibilità $(<50 \%)$, e talvolta anche da bassa specificità, si impone spesso la necessità dell'allestimento di un campione come il $\mathrm{BAL}$ raccolto con manovre invasive: risulta allora fondamentale l'affiancamento di un secondo metodo diagnostico come la RT-PCR che potrebbe aumentare notevolmente il valore clinico del risultato.

Numerosi studi hanno dimostrato l'assoluta utilità diagnostica di utilizzare la ricerca del GM e il saggio in PCR sia su siero che su BAL, nonostante performance variabili nelle diverse popolazioni a rischio $(1,8,14)$. Altri autori concludono che, in popolazioni a rischio di aspergillosi invasiva, l'esecuzione della RT-PCR su due campioni consecutivi per confermare un valore positivo aumenta soprattutto la specificità del test (dal 75\% e $87 \%$ ), mentre è sufficiente un unico campione se il risultato è negativo (alto VPN) (12).

Per quanto riguarda l'utilità dei metodi standard di diagnosi di aspergillosi invasiva, anche questo studio, sebbene gravato dal ridotto numero di campioni analizzati, conferma la bassa sensibilità sia dell'esame microscopico che dell'esame colturale. Una diagnosi microbiologica rapida (microscopica, sierologica e molecolare) può essere molto utile per un pronto intervento terapeutico, quando supportata dal sospetto clinico e/o da dati di ima-

Tabella I. Risultati su siero di GM e PCR

\begin{tabular}{lll}
\hline PAZIENTE & TEST GM & TEST PCR \\
\hline $\mathbf{l}$ & Negativo & Negativo \\
\hline $\mathbf{2}$ & Negativo & Negativo \\
\hline $\mathbf{3}$ & Negativo & Negativo \\
\hline $\mathbf{5}$ & Negativo & Negativo \\
\hline 6 & Positivo (0.90) & Negativo \\
\hline $\mathbf{7}$ & Positivo (0.50) & Positivo \\
\hline $\mathbf{8}$ & Positivo (0.5I) & Positivo \\
\hline 9 & Positivo (5.94) & Positivo \\
\hline $\mathbf{1 0}$ & Positivo (4.25) & Positivo \\
\hline & Positivo (0.70) & Negativo \\
\hline & Negativo & Negativo \\
\hline & Negativo & Indeterminato \\
\hline & Positivo $(0.74)$ & Positivo \\
\hline
\end{tabular}


ging (RX e TAC).

I dati preliminari di questo lavoro, che andranno completati con ulteriori valutazioni su un numero maggiore di campioni e di soggetti a rischio, confermano il vantaggio dell'utilizzo della RT-PCR in affiancamento al test GM su siero, per l'alta sensibilità dei test in biologia molecolare: un risultato negativo permette di escludere, con ragionevole certezza, l'infezione fungina invasiva. Anche se l'applicazione dei test in biologia molecolare su materiali delle basse vie aeree è più problematica, ed il risultato positivo di più difficile interpretazione sembra comunque importante poter disporre di un altro dato ottenuto con un metodo estremamente sensibile per significare il risultato del GM (1).

Tabella 2. Risultati su BAL dei metodi standard, sierologici e molecolari

\begin{tabular}{cccccc}
\hline \multirow{2}{*}{ PAZIENTE } & ESAME & ESAME & TEST & TEST & ESAME \\
& MICROSCOPICO & COLTURALE & GM & PCR & ISTOLOGICO \\
\hline I & Negativo & Negativo & Positivo (I.47) & Negativo & Negativo \\
\hline 2 & Negativo & Negativo & Negativo & Negativo & - \\
\hline 3 & Negativo & Negativo & Positivo (4.5) & Negativo & - \\
\hline 4 & Negativo & Negativo & Positivo (I.56) & Negativo & - \\
\hline 5 & Negativo & Negativo & Negativo & Negativo & - \\
\hline 6 & Negativo & Positivo & Positivo (5.82) & Positivo & - \\
\hline 7 & Negativo & Negativo & Positivo (5.60) & Positivo & Positivo \\
\hline 8 & Negativo & Negativo & Negativo & Negativo & - \\
\hline 9 & Positivo & Negativo & Positivo (8.44) & Negativo & - \\
\hline
\end{tabular}

\section{BIBLIOGRAFIA}

1. Avni T, Levy I, Spencher H, Yahav D, Leibovici L, Paul M. Diagnostic accuracy of PCR compared to Galactomannan in bronchoalveolar lavage fluid for diagnosis of invasive pulmonary aspergillosis: a systematic review. JCM 2012; 50(11): 3652-8.

2. Barnes RA. Early diagnosis of fungal infection in immunocompromised patients. J Antimicrob Chemother 2008 Jan; 61 (Suppl 1), Review.

3. Becker MJ, Lugtenburg EJ, Cornelissen JJ, Van Der Schee C, Hoogsteden HC, De Marie S. Galactomannan detection in computerized tomography-based broncho-alveolar lavage fluid and serum in haematological patients at risk for invasive pulmonary aspergillosis. Br J Haematol. 2003 May; 121(3): 44857.

4. Guinea J, Jensen J, Pelaez T, et al. Value of a single galactomannan determination (Platelia) for the diagnosis of invasive aspergillosis in non-hematological patients with clinical isolation of Aspergillus spp. Med Mycol 2008; 466: 575-9.

5. Guinea J, Torres-Narbona M, Gijón P, et al. Pulmonary aspergillosis in patients with chronic obstructive pulmonary disease: incidence, risk factors, and outcome. Clin Microbiol Infect. 2010 Jul; 16(7): 870-7.

6. Guinea J, Padilla C, Escribano P, et al. Evaluation of MycAssay ${ }^{\mathrm{TM}}$ Aspergillus for diagnosis of invasive pulmonary aspergillosis in patients without hematological cancer. PLoS One. 2013 Apr 19; 8(4): e61545.

7. Hoenigl M, Strenger V, Buzina W, et al. European Organization for the Research and Treatment of Cancer/Mycoses Study Group (EORTC/MSG) host factors and invasive fungal infections in patients with haematological malignancies. $J$ Antimicrob Chemother. 2012 Aug; 67(8): 2029-33.

8. Leeflang MM, Debets-Ossenkopp YJ, Visser CE, et al. Galactomannan detection for invasive aspergillosis in immunocompromized patients. Cochrane Database Syst Rev. 2008 Oct 8; (4): CD007394.
9. Marchetti O, Lamoth F, Mikulska M, Viscoli C, Verweij P, Bretagne S. European Conference on Infections in Leukemia (ECIL) Laboratory Working Groups. ECIL recommendations for the use of biological markers for the diagnosis of invasive fungal diseases in leukemic patients and hematopoietic SCT recipients. Bone Marrow Transplant. 2012 Jun; 47(6): 846-54.

10. Maertens J, Van Eldere J, Verhaegen J, Verbeken E, Verschakelen J, Boogaerts M. Use of circulating galactomannan screening for early diagnosis of invasive aspergillosis in allogeneic stem cell transplant recipients. J Infect Dis 2002 Nov 1; 186(9): 1297-306.

11. Martin-Rabadan P, Gijon P, Alonso Fernandez R, Ballesteros M, Anguita J, Bouza E. False-positive Aspergillus antigenemia due to blood product conditioning fluids. Clin Infect Dis. 2012 Aug; 55(4): e227.

12. Mengoli C, Cruciani M, Barnes RA, Loeffler J, Donnelly JP. Use of PCR for diagnosis of invasive aspergillosis: systematic review and meta-analysis. Lancet Infect Dis. 2009 Feb; 9(2): 89-96.

13. Pagano L, Akova M, Dimopoulos G, Herbrecht R, Drgona L, Blijlevens N. Risk assessment and prognostic factors for mould-related diseases in immunocompromised patients. J HYPERLINK “/I"Antimicrob Chemother 2011 Jan; 66 (Suppl 1): i5-14.

14. Pfeiffer CD, Fine JP, Safdar N. Diagnosis of invasive aspergillosis using a galattomannan assay: a metaanalysis. Clinical Infect Dis 2006 42:1417-27

15. Posterano B, Torelli R, De Carolis E, Posterano P, Sanguinetti M. Update on the laboratory diagnosis of invasive fungal infections. Mediter $J$ Hematol Infect Dis. 2011; 3(1): e2011002.

16. Singh N, Husain S. AST Infectious Diseases Community of Practice. Invasive aspergillosis in solid organ transplant recipients. Am J Transplant. 2009 Dec; 9 (Suppl 4): S180-91.

17. Sun W, Wang K, Gao W, et al. Evaluation of PCR on bronchoalveolar lavage fluid for diagnosis of invasive aspergillosis: a bivariate metaanalysis and systematic 
review. PLoS One. 2011; 6(12): e28467.

18. Tabarsi P, Soraghi A, Marjani M, et al. Comparison of serum and bronchoalveolar lavage galactomannan in diagnosing invasive aspergillosis in solid-organ transplant recipients. Exp Clin Transplant. 2012 Jun; 10(3): 278-81.

19. Thornton CR. Detection of invasive aspergillosis. $A d v$
Appl Microbiol. 2010; 70: 187-216.

20. Torelli R, Sanguinetti M, Moody A, et al. Diagnosis of invasive asper gillosis by a commercial Real-Time PCR assay for Aspergillus DNA in bronchoalveolar lavage fluid samples from high-risk patients compared to a galactomannan enzyme immunoassay. $J$ Clin Microbiol. 2011 Dec; 49(12): 4273-8. 